\title{
PREVALENCIA DE LESIONES CERVICALES NO CARIOSAS EN EL ECUADOR. UNA REVISIÓN DE LA LITERATURA.
}

\section{Prevalence of non-carious cervical lesions in Ecuador. A review of the literature.}

\author{
María Alejandra Molina Alvarado ${ }^{1}$, Mateo José Pesántez Ibarra ${ }^{1}$, Pablo Esteban Tamariz Ordoñez ${ }^{2}$. \\ ${ }^{1}$ Estudiante de la facultad de Odontología,Universidad de Cuenca.Ecuador. \\ ${ }^{2}$ Docente en la facultad de Odontología,Universidad de Cuenca.Ecuador.
}

\begin{abstract}
Resumen
Las lesiones cervicales no cariosas se caracterizan por la pérdida de estructura dentaria a nivel del límite amelocementário, sin una etiología bacteriana. Los estudios demuestran que existe un amplio rango de prevalencia de estas lesiones que pueden llegar hasta el $85 \%$, siendo significativa también en el Ecuador. El objetivo de este estudio consiste en analizar los trabajos de investigación existentes en Ecuador sobre prevalencia de lesiones cervicales no cariosas (LCNC), establecer sus resultados, analizarlos e identificar las piezas dentales más afectadas. Se realizo la búsqueda en repositorios universitarios y bases de datos como Pub Med y Elsevier para seleccionar un total de 15 publicaciones relevantes en idioma inglés y español desde el año 2010 utilizando palabras clave estratégicas.
\end{abstract}

Palabras clave: Prevalencia, Ecuador, abrasión, abfracción, erosión.

\begin{abstract}
Non-carious cervical lesions are characterized by the loss of tooth structure at the amelocemetary limit, without a bacterial etiology. Studies show that there is a wide range of prevalence of these lesions that can reach up to $85 \%$, being significant also in Ecuador. The objective of this study is to analyze the existing research in Ecuador on the prevalence of cervical non-carious lesions (NCLL), establish its results, analyze them and identify the most affected teeth. We searched university repositories and databases such as Pub Med and Elsevier to select a total of 15 relevant publications in English and Spanish since 2010 using strategic keywords
\end{abstract}

Key words: Prevalence, Ecuador, abrasion, abfraction, erosion.

\section{Introducción}

La prevalencia de las lesiones no cariosas se ha incrementado de manera importante, como consecuencia del aumento en la longevidad de la dentición. Por lo general, estas lesiones no son percibidas por el paciente, a menos que presenten sensibilidad dentinaria o compromiso estético, y se han hecho cada vez más significativas debido al incremento de las expectativas de vida y el mejoramiento en cuanto a la salud oral de la población. Según Handa A y cols, aproximadamente el $25 \%$ de la destrucción patológica de los tejidos duros del diente pueden ser atribuidos a las lesiones cervicales no cariosas, por lo que, el profesional debe realizar un diagnóstico temprano y evaluar la necesidad de tratamiento. Aunque de modo general las lesiones dentarias no cariosas no han despertado mucho interés en los clínicos e investigadores, actualmente son muy comunes en la práctica clínica, refiriéndose el incremento de las LCNC entre las afecciones estomatológicas más frecuentes. ${ }^{1,2}$

Se conoce como lesiones cervicales no cariosas al desgaste de tejido dental ubicado a nivel de la unión cemento-esmalte. Este puede ser de origen multifactorial ya sean factores patológicos, no patológicos, factores intrínsecos, o factores extrínsecos y pueden llegar a ser más frecuentes con la edad. Se clasifican en: a) Erosión o biocorrosión, que es la pérdida del tejido duro del diente causado por ácidos extrínsecos o intrínsecos, b) Abrasión, manifestada por el desgaste provocado por la fricción con un cuerpo extraño sobre la superficie dental y c) Abfracción, que consiste en una lesión que presenta una forma de cuña y es provocada por fuerzas oclusales extrínsecas que llevan a la flexión dental. ${ }^{2-4}$

El propósito de este trabajo es dar a conocer la prevalencia de LCNC de estudios publicados en Ecuador, 
cuáles son las piezas dentarias, el grupo etario y el sexo más afectado. Para que tanto pacientes como profesionales del campo odontológico sepan su importancia y se pueda realizar un buen tratamiento, evitar su progreso y pérdida de estructura dental.

Se realizó una búsqueda en repositorios universitarios del Ecuador, PubMed y Elsevier para recolectar publicaciones $^{15}$ desde el año 2010 en idioma inglés y español. Las palabras clave: prevalencia, Ecuador, abrasión, abfracción y erosión, fueron utilizadas para encontrar las referencias incluidas en esta revisión.

\section{Estado del Arte}

Se conoce como lesiones cervicales no cariosas al desgaste de tejido dental ubicado a nivel de la unión cemento-esmalte. Esta clase de lesiones tienen un origen multifactorial, y se caracterizan por la pérdida progresiva de tejido dental a nivel cervical, sin la intervención de ningún ácido de origen bacteriano. Las LCNC son una de las patologías dentales que más afecta a la población adulta. Los estudios demuestran que existe un amplio rango de prevalencia de estas lesiones que pueden ir desde el 5 al $85 \%$. Debido a la poca importancia que los pacientes le dan a esta clase de lesiones por su escasa validez estética, Las LCNC no son diagnosticadas a tiempo y conllevan a varias complicaciones como sensibilidad dental a los alimentos fríos y calientes, sensación dolorosa, exposición de la pulpa, acúmulo de placa bacteriana, irritación de la mucosa, recesiones gingivales, exposición de los cuellos radiculares y fracturas dentales. Por lo que es importante realizar un diagnóstico precoz e identificar su etiología para así poder realizar un correcto diagnóstico y tratamiento. ${ }^{2,4,5}$

Estas lesiones pueden ser causadas por factores patológicos, no patológicos, factores intrínsecos, y factores extrínsecos. La LCNC es un factor predisponente para el surgimiento de la hipersensibilidad dentinaria, ya que la exposición dentinaria que sucede después del inicio de la lesión es acompañada por la apertura de los túbulos, lo que puede evolucionar hacia una sintomatología dolorosa inicial. Caninos y premolares suelen ser uno de los dientes más afectados por ser los dientes más prominentes y proclives a tener lesiones abrasivas. ${ }^{4,6,7}$

Según Garone y Col en el 2010 se puede deducir que la gran incidencia de las lesiones cervicales se debe a los siguientes factores: región con menor área de sección transversal, menor espesor del esmalte, alineación deficiente de los prismas adamantinos, apatita más soluble. ${ }^{8}$

La literatura clasifica a las LCNC en tres tipos según su etiología y características clínicas y estas son: abrasión, abfracción y erosión.

\section{Abrasión}

La abrasión es el desgaste patológico de la estructura dentaria causada por procesos mecánicos anormales provenientes de objetos extraños o sustancias introducidas en la boca que al contactar con los dientes generan la pérdida de los tejidos duros a nivel del límite amelocementario (LAC) mediante mecanismos como pulido, frotado o raspado. ${ }^{9,10}$

Las características clínicas de esta lesión comprenden márgenes y ángulos redondeados, con contorno bien definido sin irregularidades, forma de cuña (V) o ranura, más amplias que profundas, superficies lisas y altamente pulidas, consistencia dura a la exploración, migración apical de la encía, profundidad de 1 a $2 \mathrm{~mm}$ y los dientes adyacentes suelen estar afectados. ${ }^{7}$

Una de las principales causas de esta patología es el cepillado dental, que involucra factores como la ubicación del cepillo, técnica de cepillado, longitud del mango, presión ejercida, tipo de cerda (blanda o dura), terminación de las cerdas, flexibilidad de las cerdas, flujo salival, tejido dentario involucrado, abrasividad y cantidad de la pasta. ${ }^{8,10}$

Otros hábitos pueden producir este tipo de lesión y están relacionados al trabajo del paciente, por ejemplo, interponer clavos entre los dientes y labios, uso de instrumentos musicales como la armónica o personas que trabajan con polvos abrasivos. Tratamientos dentales como el raspado y alisado radicular, el uso de prótesis removibles dentomucosoportadas podrían también ser factores causales. ${ }^{11}$

\section{Abfracción}

La abfracción es definida como un desprendimiento en forma de cuña de la estructura dental a nivel de LAC producida por fuerzas oclusales deflectivas concentradas en la región cervical, generando una flexión en el esmalte y dentina, la cual produce desprendimiento de tejidos duros del diente. Son muy comunes en superficies bucales o linguales de dientes anteriores y premolares. ${ }^{9,12}$

Se presenta en forma de cuña, es profunda, con márgenes bien definidos, presenta estrías y grietas con ángulos ásperos, puede presentarse en múltiples áreas en una pieza llegando a ser circunferencial, se puede encontrar en solo un diente y ocasionalmente la lesión, o parte de ella, puede ser subgingival. El tamaño y forma de la lesión están dados por la dirección, magnitud, frecuencia, duración y ubicación de las fuerzas que se producen cuando los dientes entran en contacto. ${ }^{7,12}$ 


\section{Erosión o biocorrosión}

Es la pérdida del tejido duro del diente causado por ácidos no producidos por bacterias, esta lesión empieza por un ablandamiento de la superficie dental que es susceptible al desgaste. La baja cantidad de saliva y el disminuido efecto buffering de la misma aumentan su incidencia. Es causada por agentes ácidos o quelantes de origen intrínseco o extrínseco. Esta lesión puede estar presente en cualquier cara del diente y depende del origen del ácido que afecta el diente, por ejemplo, se puede observar en superficies linguales, incisales y oclusales si el ácido proviene del estómago, en cambio las superficies vestibulares se encuentran afectadas por alimentos con alto contenido ácido o por aquellos provenientes del medio laboral. ${ }^{12,13}$

Factores Extrínsecos: Este tipo de ácidos vienen de diversas fuentes como medicamentos (ácido Acetilsalicílico), productos orales (pastas dentales), alimentos como jugos de frutas o la ingestión crónica de bebidas con persevantes (gaseosas). Dentro de este grupo encontramos los ácidos exógenos, que proceden de alguna ocupación, en donde los trabajadores al estar expuestos a influencia de vapores ambientales, pueden presentar lesiones corrosivas. Factores intrínsecos: Pueden ser condiciones sistémicas tales como: gastritis, síndrome de Sjörgren, ulceras gástricas, pacientes irradiados, vómitos frecuentes, trastornos alimenticios (bulimia o anorexia), embarazos. ${ }^{8}$

Estas lesiones presentan forma de "U" o de un disco poco profundo. El esmalte tiene un aspecto liso, opaco, sin decoloración, con periquematies ausentes y matriz orgánica desmineralizada y en la dentina los ácidos débiles actúan en el tejido intertubular y los ácidos fuertes en la zona peritubular, quedando aberturas en forma de embudo. ${ }^{7,12}$

Como se ha descrito anteriormente las lesiones cervicales no cariosas se han relacionado con múltiples factores como la ubicación y características del diente, dieta, propiedades de la saliva, tipo de oclusión, hábitos nocivos por parte del paciente, hábitos parafuncionales, uso de prótesis removibles, condición sistémica del paciente y las cargas funcionales que actúan sobre el diente. Además, se menciona una relación entre las LCNC y los factores sociodemográficos, edad, técnica de cepillado, tipo de cepillo, y componentes abrasivos presentes en la pasta dental. ${ }^{15}$

A pesar de que se han descrito distintos factores causales para las LCNC la etiopatogenia no es clara hasta la actualidad y se atribuye a diferentes mecanismos multifactoriales. La pérdida de estructura dentaria a nivel cervical puede ser causada por abrasión, erosión y abfracción, o por mecanismos combinados. ${ }^{14,15}$

La idea de una etiología combinada, multicausal o multifactorial fue ganando terreno y se describen lesiones combinadas a predominancia de uno u otro factor. Pocas veces estas lesiones, como el caso de la abfracción se encuentran actuando solas, por lo general estas lesiones son comunes de encontrar en forma combinada, por ejemplo, la erosión por consumo de comidas o bebidas muy ácidas, seguida por la abrasión producto de un cepillado inmediato después del ataque ácido, causando una lesión a nivel cervical sobre todo si la técnica de cepillado es incorrecta o si la pasta dental es muy abrasiva. ${ }^{15}$

En algunas ocasiones estas lesiones pueden presentarse por dos causas en un mismo individuo y estar relacionadas entre sí; incluso pueden combinarse generando lesiones mixtas en un mismo diente. Se pueden mencionar las siguientes combinaciones: atrición - abfracción, atrición abrasión, atrición - erosión, abfracción - erosión, abrasión erosión, abfracción - abrasión y abfracción - biocorrosión según los mecanismos causales que actúen. ${ }^{9}$

Las LCNC son consideradas un problema creciente en la práctica de la estomatología restauradora, por su importancia en esta área se enseñará los resultados de algunos trabajos sobre prevalencia en el Ecuador para demostrar su frecuencia y las piezas más afectadas por estas lesiones.

Un estudio realizado a pacientes que acudieron a la Universidad Católica de Santiago de Guayaquil en el año 2016 revelo que de los 100 individuos que conformaron la muestra, 28 pacientes $(28 \%)$ presentaron por lo menos una lesión cervical no cariosa. En cuanto a la prevalencia de LCNC según el género, se pudo observar 19 de los 50 individuos pertenecientes al género femenino presentaron lesión cervical no cariosa (38\%); mientras que 9 de los 50 pacientes masculinos, presentaron dicha lesión (18\%). Se vio mayor frecuencia pasados los 40 años de edad representado por el $42 \%$. El primer premolar superior derecho y el primer premolar inferior izquierdo fueron las piezas más afectadas. ${ }^{2}$

En otro estudio realizado por la misma universidad se estableció una prevalencia de $76.6 \%$ de una muestra de 120 personas. Según el género, se pudo observar que 31 pacientes de género masculino (34\%) se encontraban afectados, en contraste, el género femenino se encontró afectado en un $66 \%$. El rango de edad de 37-47años resulto ser el más afectado siendo el $30 \%$ de la muestra. De manera general y porcentual las lesiones más comunes halladas fueron las abfracciones (39\%), abrasiones $(37 \%)$ y las erosiones con un $24 \% .^{4}$

Un tercer estudio realizado por Janine Ordoñez 
estudiante de esta misma institución en el año 2012 demostró un $65 \%$ de prevalencia de LCNC de una muestra de 100 pacientes. En este estudio de 9 pacientes hombres 6 resultaron afectados $(66.6 \%)$ y de las 91 mujeres que conformaron la muestra 49 presentaban LCNC (53.8\%). El rango de edad más afectado en este estudio estuvo comprendido entre 26-40 años de edad que represento el $49.32 \%$. De 2793 dientes examinados 221 presentaron al menos una LCNC y de estos, 95 fueron premolares (43\%) siendo el grupo dentario más afectado. La lesión abrasiva fue la más prevalente representada por el $27.7 \%{ }^{9}$

Melissa Bajaña $\mathrm{R}$ en su estudio publicado en el año 2020 establece que de una muestra de 100 individuos el $55 \%$ presento LCNC; en cuanto al género se observó que el masculino superó al femenino con un $29 \%$ de presencia de LCNC. Dentro del grupo etario podemos encontrar que el mayor porcentaje de LCNC se encontró en el grupo de 41 a 60 años de edad con un $45 \%$. Además, concluyó que el grupo dentario más afectado eran los premolares. ${ }^{16}$

En un estudio realizado en la universidad Central del Ecuador en el año 2017 por Karla Moreno L con una muestra de 120 pacientes se estableció que la prevalencia general de LCNC fue el $98.3 \%$. En cuanto al género de las 78 mujeres incluidas $65 \%$ presentaron estas lesiones y de los 42 hombres el $35 \%$ las presentaron; el grupo etario más afectado estuvo comprendido entre 40-50 años. El diente más afectado fue el primer premolar inferior representando el $12.5 \%$ y la lesión más frecuente fue la abfracción. ${ }^{8}$

Otro estudio realizado en 152 pacientes que acudieron a la Clínica Integral de la Facultad de Odontología de la Universidad Central del Ecuador en el 2016. Presentó una prevalencia de LCNC de un $42,1 \%$ en total; se presentó mayor prevalencia en varones que en mujeres. En edades desde los 45 años o más se presentó la mayoría de estas lesiones y las más frecuentes fueron las abfracciones comparadas con abrasiones. ${ }^{17}$

En la universidad San Gregorio de Portoviejo en el año 2014 un estudio realizado por Mabel Macias M demostró que de una muestra de 180 personas estuvieron afectadas con LCNC 48, representado por el 26\%; 165 fueron mujeres y de estas 41 presentaron estas lesiones ( $25 \%$ ), los hombres estudiados fueron 15 de estos el $53 \%$ presento LCNC. El grupo etario más afectado fue el de mayores a 55 años, la pieza más afectada fue el 1er premolar $(31 \%)$ y la lesión más frecuente es la abfracción $48 \%{ }^{6}$

En el centro de salud Andrés de Vera del cantón Portoviejo, provincia de Manabí, fueron examinados 80 pacientes, 55 fueron diagnosticados con LCNC lo que equivale al $69 \%$; 13 pacientes fueron del sexo masculino lo que equivale a un $24 \%$ y 42 fueron del sexo femenino lo que equivale a un $76 \%$. Según la edad se observa que de los 55 pacientes diagnosticados con LCNC las edades entre 26-49 años equivalió a un $51 \%$ y entre 5070 años a un $49 \%$. Además, la abrasión equivalió a un $24 \%$ siendo la más prevalente. $^{7}$

En el año 2017, un estudio realizado en la UDLA por David Caicedo P en pacientes de 18 a 40 años demostró que del total de 380 pacientes revisados el $45.3 \%$ presentó algún tipo de lesión cervical no cariosa. En lo referente a la abrasión, se determinó que es más prevalente en personas de género femenino, las abfracciones tienen mayor prevalencia en las personas de género masculino. De acuerdo al estudio la pieza dental más afectada por estas lesiones son las piezas $1.4 ; 2.4$ y 3.4. Además, se demostró que la LCNC con mayor prevalencia es la abrasión con un $57 \% .^{18}$

Un estudio realizado en el 2014 por Daniela Diaz M en la universidad Santiago de Guayaquil en 108 pacientes revelo que el $19 \%$ presentaron LCNC. La pieza más afectada fue la $4.4(14.63 \%){ }^{19}$

En el año 2020 Erika Vega C. estudiante de la Universidad Central del Ecuador demostró en su estudio de 362 pacientes que la prevalencia de LCNC fue del 72,4\%, en su mayor parte se presentó en el sexo femenino (46,1\%). La tasa más alta de LCNC se registró en el grupo de edad de 55-64 años. ${ }^{12}$

En la facultad de Odontología de la Universidad de Cuenca durante el periodo marzo - agosto 2015, de un total de 105 estudiantes siendo 63 mujeres y 42 hombres de 18 y 26 años, se pudieron encontrar los siguientes resultados: 65 estudiantes de los 105 analizados presentaron LCNC que corresponde a un porcentaje de $61,9 \%$. Los premolares fueron las piezas con mayor frecuencia de LCNC con un porcentaje de $63,5 \%$. En cuanto a la forma y clasificación de las LCNC encontradas la más prevalente fueron las abrasiones con un $95,3 \% .^{13}$

\section{Discusión}

La prevalencia de LCNC en el Ecuador ha sido ampliamente estudiada y diversos resultados han sido publicados. Se ha observado que las LCNC cada vez se presentan con más frecuencia sobre todo en pacientes de edad adulta los cuales son más propensos y las prevalencias pueden variar en rangos altos.

Los rangos de prevalencia entre los 12 estudios observados son amplios siendo la menor prevalencia la observada en el estudio de Daniela Diaz M. en el año 2014 en la universidad Santiago de Guayaquil representando el 


\begin{tabular}{cccc}
\hline Institución & Año & Muestra & \% LCNC \\
\hline Universidad Católica de Santiago de Guayaquil & 2016 & 100 & $28 \%$ \\
Universidad Católica de Santiago de Guayaquil & 2017 & 120 & $76.6 \%$ \\
Universidad Católica de Santiago de Guayaquil & 2012 & 100 & $65 \%$ \\
Universidad Católica de Santiago de Guayaquil & 2020 & 100 & $55 \%$ \\
Universidad Católica de Santiago de Guayaquil & 2014 & 108 & $19 \%$ \\
Universidad Central del Ecuador & 2017 & 120 & $98.3 \%$ \\
Universidad Central del Ecuador & 2016 & 152 & $42.1 \%$ \\
Universidad Central del Ecuador & 2020 & 362 & $72.4 \%$ \\
Universidad San Gregorio de Portoviejo & 2014 & 180 & $26 \%$ \\
Universidad San Gregorio de Portoviejo & 2015 & 80 & $69 \%$ \\
Universidad de las Américas & 2017 & 380 & $45.3 \%$ \\
Universidad de Cuenca & 2015 & 105 & $61.9 \%$ \\
\hline
\end{tabular}

Tabla 1. Resumen de prevalencias.

$19 \%$, lo cual difiere con la mayor prevalencia de $98 \%$ en un estudio realizado en la universidad Central del Ecuador en el año 2017 por Karla Moreno L. El promedio de las prevalencias presentadas en todos los estudios es de $54.8 \%$.

Por otro lado 8 de los 12 estudios analizados establecieron cual fue el grupo dentario más afectado, coincidiendo que es el grupo premolar, lo que concuerda con el estudio publicado por Rodríguez Chala en el año 2002 en el cual el premolar estuvo afectado en un $55 \%$. En el 2013 J. Segura realizó un estudio en la Universidad Nacional Mayor de San Marcos en Perú, en el cual estableció de igual manera a los premolares como los dientes más afectados. En contraste en el 2000 D. Telles y cols indicaron que los primeros molares inferiores eran los más afectados ya que representaban el $21.3 \%{ }^{16,18}$

En cuanto al género, de los 12 estudios contemplados en este artículo 9 indicaron la prevalencia de LCNC de acuerdo al sexo, de estos, 5 indicaron que se presentaba más en mujeres y en los otros 4 fueron los hombres los más afectados. Concordando con esto está la publicación realizada en la Facultad de Odontología de la Universidad Autónoma del Paraguay en el 2008 donde se encontró una prevalencia mayor en el género femenino (51\%). Esto difiere de un estudio realizado por Barranca en el 2004 en el cual la mayor prevalencia se dio en el género masculino representado por el $65.07 \% .^{17,20}$

El tipo de LCNC más prevalente fue indicada en 8 de los 12 estudios, de esta cantidad 4 indicaron a la abrasión como la lesión más prevalente y los otros 4 a la abfracción. Esto concuerda con un estudio realizado en el 2013 en Facultad de Odontología de la Universidad Nacional Mayor de San Mateo, en donde se indicó que la abrasión era la
LCNC más común. ${ }^{8}$

Un estudio realizado por Beltrán E en la Universidad Católica de Santiago de Guayaquil en el 20017 en 100 individuos entre 18 y 65 años encontró que la edad en la que se presentó con mayor frecuencia las LCNC fue en las edades superiores a 40 años representado por el $42 \%$, lo que concuerda con un estudio realizado en la misma universidad en el 2020 por Bajaña M en 100 pacientes entre 18 y 60 años en donde los más afectados estuvieron entre el rango de 41 a 60 años con el $45 \%$. De la misma manera otro estudio del 2017 realizado en la universidad Central del Ecuador por Moreno K. establece que los pacientes que más presentan LCNC está comprendido entre 40 y 59 años en un $80 \%$. Como podemos observar la presencia de LCNC está directamente relacionada con el aumento de la edad. ${ }^{2,8,16}$

\section{Conclusiones}

- La prevalencia en el Ecuador es muy variada, va desde porcentajes de $19 \%$ al $98 \%$ y se obtuvo un promedio de $54.8 \%$ de los resultados de los 12 estudios.

- Se estableció que el grupo dentario más afectado es el grupo premolar.

- $\quad$ El sexo más afectado por las LCNC es el femenino.

- Dentro de los 12 estudios analizados 4 apoyan a las abrasiones como las LCNC más prevalentes y otros 4 establecen a las abfracciones como las más frecuentes.

- Se ha visto una asociación directa entre el aumento de la edad con la mayor prevalencia de LCNC.

- La etiopatogenia no es clara hasta la actualidad y se puede atribuir a diferentes mecanismos multifactoriales por lo que se debería seguir haciendo investigaciones o seguimiento al respecto para establecer de manera correcta el diagnóstico y tratamiento de las LCNC.

\section{Referencias}

1 Ceballos Rojas MA, Abad Sastre A. Prevalencia de las lesiones no cariosas cervicales en pacientes atendidos en la Clínica Estomatológica “Juan Manuel Márquez.” 16abril. 2019;58(273):73-6.

2 Beltrán Gordón E, Mosquera Chávez T. Prevalencia de lesiones cervicales no cariosas en pacientes de la Clínica UCSG semestre B-2016. Repos Digit UCSG. 2017.

3 Grippo JO, Simring M, Coleman TA. Abfraction, abrasion, biocorrosion, and the enigma of noncarious cervical lesions: A 20-year perspective [Internet]. 
Vol. 24, Journal of Esthetic and Restorative Dentistry. 2012 [cited 2020 Oct 30]. p. 10-23. Available from: http://doi.wiley.com/10.1111/j.1708-8240.2011.00487.x.

4 Hidalgo Neira R, Mosquera Chávez T. Prevalencia de lesiones cervicales no cariosas en pacientes de la Clínica UCSG semestre A-2017. Repos Digit UCSG. 2017.

5 Zucchelli G, Gori G, Mele M, Stefanini M, Mazzotti C, Marzadori M, et al. Non-Carious Cervical Lesions Associated With Gingival Recessions: A DecisionMaking Process. J Periodontol [Internet]. 2011 Dec [cited 2020 Oct 30];82(12):1713-24. Available from: http://doi.wiley.com/10.1902/jop.2011.110080.

6 Cabrera Sánchez M, Macías Madrid MM. Lesiones 16 cervicales no cariosas en los pacientes de 15 a 70 años de edad, atendidos en el Centro de Salud San Pablo del cantón Portoviejo en el período marzo - agosto de 2014. [Internet]. Universidad San Gregorio de Portoviejo; 2014. Available from: http://repositorio.sangregorio.edu.ec/handle/123456789/223.

7 Vera Loor PA. Lesiones cervicales no cariosas en pacientes que acuden al centro de Salud Andrés de Vera en el Cantón Portoviejo de la Provincia de Manabí de la República del Ecuador periodo marzo - junio de 2015 [Internet]. Universidad 18 San Gregorio de Portoviejo; 2015. Available from: http://repositorio.sangregorio.edu.ec/handle/123456789/656.

8 García Merino IR, Moreno León KE. Prevalencia de las lesiones cervicales cariosas y no cariosas en pacientes que acuden a la clínica de tercer nivel del facultad de Odontología de la universidad central del Ecuador. [Internet]. 19 Universidad Central del Ecuador; 2017. Available from: http://www.dspace.uce.edu.ec/handle/25000/12514.

9 Ordóñez García JE. Prevalencia de lesiones cervicales no cariosas en pacientes de 18 a 60 años que asistan al Servicio Odontológico del Hospital Teófilo Dávi- 20 la en el mes de Junio 2012 [Internet]. Universidad Católica de Santiago De Guayaquil; 2012. Available from: http://repositorio.ucsg.edu.ec/handle/3317/874.

10 Milosevic A. Abrasion: A Common Dental Problem Revisited. Prim Dent J [Internet]. 2017 Mar 1 [cited 2020 Oct 30];6(1):32-6. Available from: http://journals.sagepub.com/doi/10.1177/205016841700600104.

11 Arteaga Andrade ME. Caracterización de las lesiones no cariosas en zona cervical de pacientes adultos [Internet]. Universidad Laica Eloy Alfaro de Manabí; 2017. Available from: http://repositorio.uleam.edu.ec/handle/123456789/315

12 Rueda Landázuri WG, Vega Cañizarez EL. Prevalencia y factores asociados a lesiones cervicales no cariosas en pacientes que acuden a la Clínica Integral de la Facultad de Odontología de la Universidad Central del Ecuador [Internet]. Universidad Central del Ecuador; 2020. Available from: http://www.dspace.uce.edu.ec/handle/25000/21423.

13 Silva Ulloa S. Frecuencia de las lesiones cervica- les no cariosas en los estudiantes de la facultad de odontología de la universidad de Cuenca. [Internet]. Universidad de Cuenc; 2015. Available from: http://dspace.ucuenca.edu.ec/handle/123456789/24059

14 Calabria Díaz H. Lesiones no cariosas del cuello dentario: patología moderna, antigua controversia. Scielo [Internet]. 2009;11(12). Available from: http://www.scielo.edu.uy/scielo.php?script=sci_arttext\&pid=S168893392009000100003

15 Navarrete MJ. Etiopatogenia de lesiones cervicales no cariosas. [Internet]. Universidad de Guayaquil; 2020. Available from: http://repositorio.ug.edu.ec/handle/redug/49712

6 Bajaña Rivadeneira MN, Altamirano Vergara NM. Prevalencia de lesiones cervicales no cariosas y presencia de hipersensibilidad. UCSG, semestre B-2019. Repos Digit UCSG [Internet]. 2020; Available from: http://repositorio.ucsg.edu.ec/handle/3317/14250

7 Balseca Ibarra EG, Sepúlveda Amar SG. Prevalencia de lesiones cervicales no cariosas en pacientes de la clínica de la Facultad de Odontología de la Universidad Central del Ecuador. Repos Digit Univ Cent del Ecuador [Internet]. 2017; Available from: http://www.dspace.uce.edu.ec/handle/25000/9098

18 Caicedo Proaño DA. Prevalencia de lesiones clase $\mathrm{V}$ no cariosas en pacientes de 18 a 40 años que acuden al centro de atención odontológica UDLA en el periodo septiembre -octubre 2017 [Internet]. Universidad de las Américas; 2018. Available from: http://dspace.udla.edu.ec/handle/33000/8446

19 Díaz Maldonado DM. Prevalencia de lesiones cervicales no cariosas y su grado de desgaste en alumnos de odontología de la UCSG [Internet]. Universidad Católica de Santiago de Guayaquil; 2014. Available from: http://repositorio.ucsg.edu.ec/handle/3317/1900

0 Barranca A, Lara E, González E. Desgaste dental y bruxismo. ADM. 2004;61(6):215-9.

Recibido: 29 de diciembre de 2020

Aceptado: 19 de febrero de 2021 